\title{
Design and Application of Magneto-Rheological Fluid "MRF"
}

\author{
A. G. Olabi and A. Grunwald \\ Dublin City University, School of Mechanical and Manufacturing Engineering, \\ Glasnevin, Dublin 9, Ireland, Email: abdul.olabi@dcu.ie
}

\begin{abstract}
:
Magneto-Rheological Fluid (MRF) technology is an old "newcomers" coming to the market at high speed. Various industries including the automotive industry are full of potential MRF applications. Magneto-Rheological Fluid technology has been successfully employed already in various low and high volume applications. A structure based on MRF might be the next generation in design for products where power density, accuracy and dynamic performance are the key features. Additionally, for products where is a need to control fluid motion by varying the viscosity, a structure based on MRF might be an improvement in functionality and costs. Two aspects of this technology, direct shear mode (used in brakes and clutches) and valve mode (used in dampers) have been studied thoroughly and several applications are already present on the market. Excellent features like fast response, simple interface between electrical power input and mechanical power output, and precise controllability make MRF technology attractive for many applications.
\end{abstract}

This paper presents the state of the art of an actuator with a control arrangement based on MRF technology. The study shows that excellent features like fast response, simple interface between electrical power input and the mechanical power output, and controllability make MRF the next technology of choice for many applications.

\section{Keywords:}

Magneto-Rheological Fluid, Actuator, Valve mode, Shear Mode, Squeeze mode. 


\section{Introduction:}

Ever since some industrial issues were solved, both the technical and the commercial benefits for various MRF applications have become very promising. As a result MRF development is on going continuously. Approximately sixty years ago, in the 1940s, Jacob Rabinov discovered the MRF effect at the US National bureau of Standards. At the same time W. Wislow was working on a competitive technology called ElectroRheological Fluid (ERF). Since the time when both technologies were discovered in the 1940s, more research work has been carried out on ERF than on MRF. There are some similarities between the two different technologies regarding the required power, but in the case of ERF, thousands of volts and some milli-amperes are required, and in the case of MRF, normally between 2 and 24 volts and some amperes are required. The electro-rheological (ER) effect depends on an electrostatic field and the magnetorheological (MR) effect depends on a magnetic field. MRF products have between 20 and 50 times higher control effect than the equivalent ERF products. Also with MRF technology today there is better stability with regard to contaminants. All these MRF technology advantages have created a very high level of interest to introduce products based on MRF technology during the most recent couple of years. Table 1 gives an overview of the ER and MR key features [1].

Table 1, MRF versus ERF [1-5]

\begin{tabular}{|l|c|c|}
\hline Representative Feature & MRF & ERF \\
\hline Max. Yield Stress & $50-100 \mathrm{kPa}$ & $2-5 \mathrm{kPa}$ \\
\hline Power Supply & $2-24 \mathrm{~V} @ 1-2 \mathrm{~A}$ & $2-5 \mathrm{kV} @ 1-10 \mathrm{~mA}$ \\
\hline Response time & some millisecond & some millisecond \\
\hline Operational Field & $\sim 250 \mathrm{kA} / \mathrm{m}$ & $\sim 4 \mathrm{kV} / \mathrm{mm}$ \\
\hline Energy density & $0.1 \mathrm{~J} / \mathrm{cm}^{3}$ & $0.001 \mathrm{~J} / \mathrm{cm}^{3}$ \\
\hline Stability & Good for most impurities & Poor for most impurities \\
\hline Operational temperature & $-40^{\circ} \mathrm{C}$ up to $+150^{\circ} \mathrm{C}$ & $-25^{\circ} \mathrm{C}$ up to $+125^{\circ} \mathrm{C}$ \\
\hline
\end{tabular}


Over approximately the most recent five years more MRF publications than ERF publications have been presented in the public domain. At the beginning of the development work on MRF, non-predictable behaviour, such as in-use thickening, sedimentation and abrasion [2,3] were described. This created some challenges for the industrialization of the first application based on MRF, especially for an automotive application. During the most recent few years the stability, sedimentation and abrasive behaviour have been studies in several universities and companies in the USA (Lord Inc.), in Europe (DEA, BASF, Bayer) and in Japan (Bridgestone Inc., Sigma Inc.) Recently MRF applications such as dampers, clutches, active bearings have already come to the market or are close to the start of serial production.

\section{Rheological Background to the MRF Technology}

Rheology is the study of flow and deformation. Flow capability and deformation, which is either elastic or plastic, have common features and the study of both subjects must overlap. In a conventional application with a conventional liquid i.e. a hydraulic pump or a damper, the most important property is the viscosity and viscosity changes with temperature. Because of this, temperature would normally be considered as an uncontrollable feature. There are two ways of expressing the viscosity - dynamic and kinematic viscosity. Dynamic viscosity is defined by:

$$
\eta=\frac{\tau}{\gamma}, \text { with } \tau=\text { shear stress }\left(\frac{N}{m^{2}}\right), \gamma=\text { shear rate }\left(\frac{1}{s}\right), \eta(P a \cdot s)
$$

Kinematic viscosity is defined by:

$$
u=\frac{\eta}{\rho}, \text { with } \rho=\operatorname{desity}\left(\frac{\mathrm{kg}}{\mathrm{m}^{3}}\right), \eta(\mathrm{Pa} \cdot \mathrm{s}), v\left(\frac{\mathrm{m}^{2}}{\mathrm{~s}}, 10^{4} \text { Stokes, } 10^{6} \mathrm{cSt}\right)
$$

The temperature dependency of a conventional fluid i.e. silicon oil or a mineral oil is determined by the approximation:

$$
\eta(T)=A \cdot e^{\left(\frac{b}{(T+273)}\right)}
$$


The factors A and b are experimentally defined for specific liquids. Newton evaluated the relationship between shear stress and shear rate for various materials. In the case of water this relationship is linear. The common name for such a fluid is a Newtonian fluid. For a Newtonian fluid the dynamic viscosity has a constant value. Typical relationships between shear rate and shear stress, and the corresponding relationships between dynamic viscosity and shear stress for various fluids are shown in Figure 1. In addition to the graphical representation, equations have been developed to quantify the relationships between these rheological parameters.
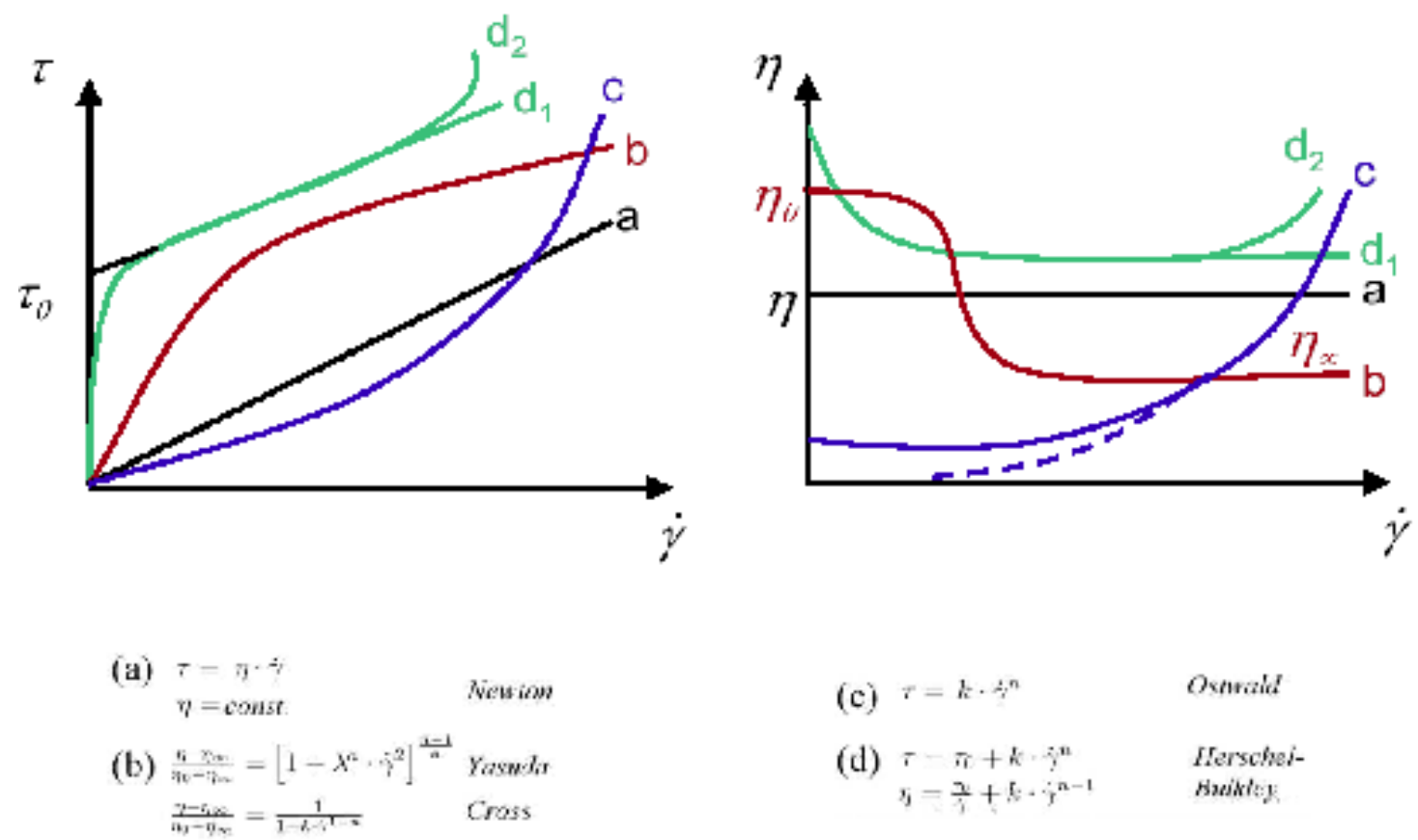

Fig. 1, Different types of fluids with equations [11]

The relationship between the shear stress, viscosity and shear rate depends on the fluid type. In the case of the black curve marked with the small letter (a), the fluid can be recognised as a Newtonian fluid. The viscosity does not change despite different shear rate values, and the shear stress has a linear relationship with the shear rate. The representative fluid with this behaviour is water. In the cases of curves (b) and (c), the shear stress has a reducing (b) or increasing (c) dependency with shear rate. There is an analogous relationship between viscosity and shear rate, which corresponds to each of these fluid behaviours. The representative fluids with these behaviours are ketchup tooth pasts, etc. The curve (d) describes behaviour, which is close to the behaviour of 
MRF. The rheological behaviour of MR fluids, where no magnetic field is present, is very similar to the pattern of the carrier fluids, except that the metal powder content of the MR fluid makes the liquid slightly "thicker”[1,2,4]

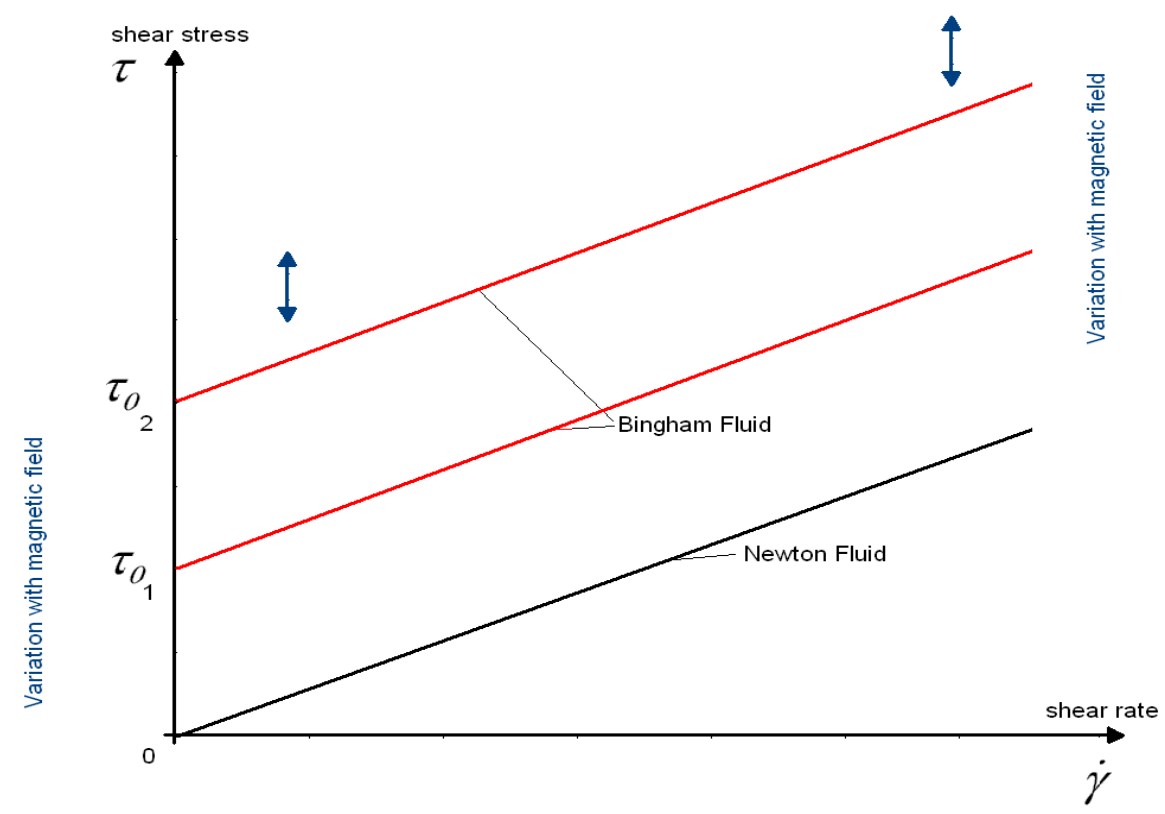

Fig. 2, Shear stress versus speed for Newton and Bingham model of MRF

Figure 2 shows the typical relationship between shear stress and shear rate for a Bingham fluid and compares this with a Newtonian fluid. It is recognised that the Bingham model is valid for use in describing the rheological features of an MR fluid $[1,2,4]$. When a magnetic field is not present an MR fluid behaves like a Newtonian fluid. When a magnetic field is present, the MR fluid shows a characteristic of Bingham fluids. At zero shear rate there is some resistance to flow. The force causes a plastic deformation, but there is no continuous movement. In this condition, the maximum stress, which can be applied without causing continuous movement, is the yield stress and this is a function of the magnetic field strength. For an MR fluid, the yield stress can be controlled, increasing or decreasing with the strength of the magnetic field as described in Eq. (4).

$$
\tau=\tau(H)+\eta \cdot \gamma
$$

In a magnetic field each metal particle becomes a dipole (North and South) and there is a tendency for a chain to be created with neighbouring particles. The chains have a mechanical resistance to the fluid flow, and because of this the viscosity of the fluid 
increases. In the magnetic field, the particle chains are structured according to the pattern of magnetic flux paths. The mechanical resistance to flow of this chain structure can be controlled by the magnetic field strength and results in viscosity changes from free flowing liquid to a semi-solid condition. The MR effect is reversible. When the magnetic field is removed, the original condition of the liquid is re-established. The magnetic field controls the level of the MR effect. The magnetic field required to impose this control depends on the fluid formulation, especially on the quality and quantity of the metal powder. To increase the yield stress capability it is necessary to have a higher percentage of metal powder and larger particles to support the chain structure. The yield stress capability also depends on the magnetization characteristics of the particles. This magnetization is expressed as the Flux density, B (Tesla) as it varies with the magnetic field strength, $\mathrm{H}(\mathrm{A} / \mathrm{m})$. It depends on the concentration of metal powder in the liquid and on the actual nature of the material of the particles. MR fluids based on carbonyl iron are capable of operating with yield stresses of $100 \mathrm{kPa}$. In order to have a predictable behaviour it is recommended the equipment should be operated in the linear section of the $B=f(H)$ curve, and it is better if the hysteresis effect is very small. Detailed design must manage a trade-off between mechanical, magnetic and rheological factors.

Depending on the fluid flow and on the rheological stress, there are three different modes of MRF operation: the Direct Shear Mode, the Valve Mode and the Squeeze Mode. The basic working principles of each of these are shown in Table 2.

Table 2, MRF operational modes

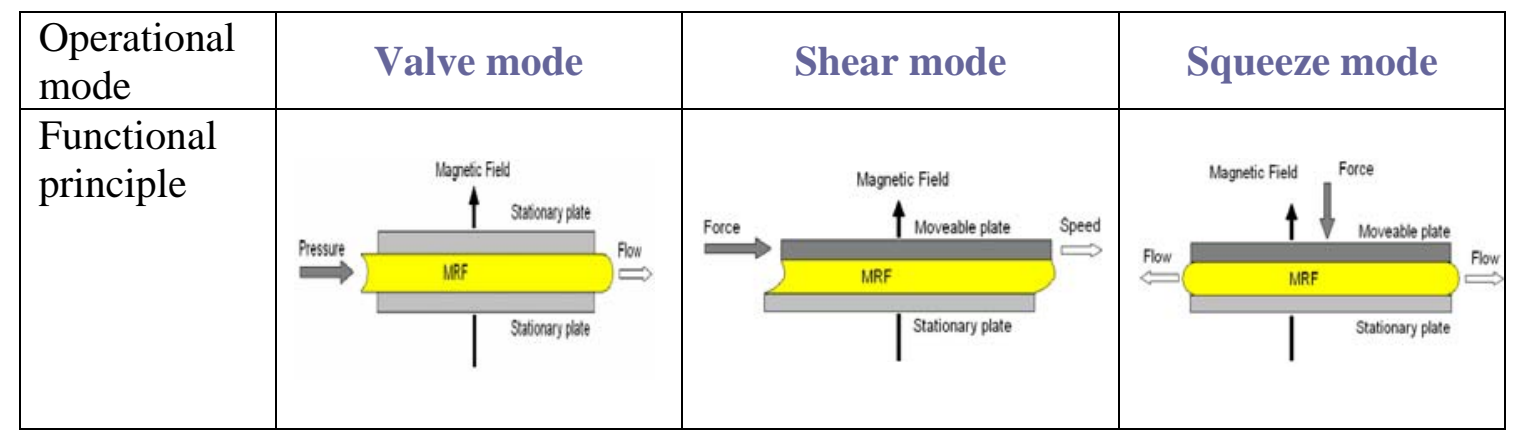




\section{MRF Components:}

A Magneto-Rheological-Fluid is a fluid with rheological behaviour which depends on the strength of a magnetic field. The rheological status changes reversibly from liquid to the solid. The Greek word "rheos" means flowing and rheology is the science of deformation behaviour of materials which are able to flow. Normally the rheological property of viscosity changes with other physical properties, such as chemical composition, shear stress and temperature. These features are not easily controlled in most applications because they are fixed by the environment in a particular situation. In the case of all fluids the variation of viscosity with temperature is reversible but this does not allow the viscosity to be controlled easily. In the case of MR the fluid viscosity becomes intelligently controllable using the magnetic field. This change of viscosity up to the solid condition is reversible and is the basic feature of MRF technology. The MRF effect is the difference in rheological properties with and without a magnetic field.

There are basically three components in an MR fluid: basic fluid, metal particles and stabilizing additives [1-16].

The Base fluid has the function of the carrier and naturally combines lubrication (in combination with additives) and damping features. For the highest MRF effect the viscosity of the fluid should be small and almost independent of temperature. In this way the MRF effect will be the dominant effect when it is compared with the natural physical viscosity varying with temperature and shear stress. Basically in the off-state (without any magnetic effects) MR fluids behave like the base fluid in accordance with their chemical compositions. There are different types of liquid which can be used as the carrier fluid i.e. hydrocarbon oils, mineral oils or silicon oils. As with any type of particle suspended in a fluid, the base fluid will have a higher viscosity when the concentration of metal particles is very high. The fluid will appear to be "thicker" [5]. So even in the off-state, the fluid with the powder will have an increased viscosity. Usually the dynamic viscosity $\eta_{\mathrm{d}}$ at ambient temperature is around $100 \mathrm{mPa}$ [8]. 
In the on-state (with a magnetic field in place) the Metal particles are guided by the magnetic field to form a chain-like structure. This chain-like structure restricts the motion of the fluid [4] and therefore changes the rheological behaviour of the fluid. The MR-effect is produced because of this resistance to flow caused by the chain-like structure. The metal particles are usually made of carbonyl iron, or powder iron, or iron/ cobalt alloys to achieve a high magnetic saturation. The amount of metal powder in MRF can be up to $50 \%$ by volume [1-16]. The particle size is in the $\mu$-meter range and varies depending on the manufacturing processes. The particle size can be chosen to achieve various purposes. In the case of carbonyl iron the particle size ranges between 1-10 $\mu$-meter. Larger particles and higher fractions of powder in the MR fluid will provide higher torque in the on-state, but at the same time the viscosity of the MR fluid in the off-state will also be higher under these conditions. The material specification, especially the permeability is also a very important factor for controlling the MR-effect.

The additives include stabilizers and surfactants [7]. Additives are suspending agents, thixotropes, friction modifiers and anti-corrosion/wear components. Highly viscous materials such as grease or other thixotropic additives are used to improve settling stability [13]. Ferrous naphthanate or ferrous oleate can be used as dispersants and metal soaps such as lithium stearate or sodium stearate as thixotropic additives [14]. Additives are required to control the viscosity of the liquid and the settling rate of the particles, the friction between the particles and to avoid the in-use thickening for a defined number of off-duty cycles.

All three components define the magneto-rheological behaviour of the MR fluid. The total density depends on the formulation and is approximately by $3-4 \mathrm{~g} / \mathrm{cc}$. The change of one of the MRF components will lead to rheological changes (in the off-state) and to magneto-rheological changes in behaviour (in the on-state) . Finally a trade-off between the achievable performances of all three components in combination is required in order to optimise a formulation.

There are some similarities between $\mathbf{M R}$ fluids and Ferrofluids. The magnetorheological behaviours of the two types of fluid are different because there is a difference in both the quality and the quantity of the metal powders. Common to both is that they contain iron particles, a basic fluid and additives. The main difference is 
the size, the quantity and the quality of the iron particles. In the case of MR fluids the iron particles are large, larger than $1 \mu \mathrm{m}$. In the case of Ferrofluids the iron oxide particles are much smaller, about 30nm [5]. With MRF there is a change of state from liquid to solid when a magnetic field is switched on, whereas a Ferrofluid remains liquid even in a high magnetic field [5]. In the Ferrofluid effect the strong yield stress behaviour is almost nonexistent, whereas it is the ability to create the chain structure in MR fluids with mechanical resistance to flow which is of paramount importance in the MRF effect. Carbonyl iron based MR fluids are able to develop yield stresses of $100 \mathrm{kPa}$, but a typical yield stress for a Ferrofluid is $10 \mathrm{kPa}$. The viscosity dependency due to a magnetic field in a Ferrofluid is a secondary effect. The main Ferrofluid effect is to guide and attract the fluid according the magnetic field intensity.

Ferrofluids are very stable due to the particle sizes. The particles are also less abrasive than is the case for MR fluids.

The above description indicates the differences between the two types of fluid in terms of functionality. It is also possible to define the difference more exactly in terms of an energy factor $\lambda$. This is defined for an individual particle in terms of Brownian thermal energy and magnetic polarisation energy as follows:

$$
\lambda=\frac{\mu_{0} \cdot P_{m a g}^{2} \cdot V}{(12 \cdot k \cdot T)}
$$

In this equation: $P_{\text {mag }}$ is the polarization, $V$ is the particle volume, $k$ is Bolzmann's constant and $T$ is the temperature. In the case where the energy factor $\lambda$ is larger than 1 , the magnetization energy is larger than the thermal energy and the fluid will have the MRF functionality in an applied magnetic field. Otherwise the thermal energy is larger and the magnetic field would just guide the particles according to the flux density. An overview of representative features is shown in Table 3. 
Table 3, MRF versus Ferrofluid [1-5]

\begin{tabular}{|l|c|c|}
\hline Representative Feature & MRF & Ferrofluid \\
\hline Energy factor $\lambda$ & $>1$ & $10 \mathrm{kPa}$ \\
\hline Max. Yield Stress & $100 \mathrm{kPa}$ & some $n \mathrm{~m}$ \\
\hline Particle size & some $\mu \mathrm{m}$ & iron oxide \\
\hline Particle material & carbonyl iron & up to $10 \%$ \\
\hline Fraction by volume & up to $50 \%$ & good \\
\hline Stability & medium & Controllable liquid flow \\
\hline Functionality & Controllable shear stress & \\
\hline
\end{tabular}

\section{MRF Operational Modes and Applications:}

Depending on the fluid flow and the rheological stress there are three different modes of MRF operation: Direct Shear Mode, Valve Mode and Squeeze Mode.

\subsection{Valve Mode:}

The valve mode as an operational mode is used in dampers, shock absorbers and is shown schematically in Figure 3.

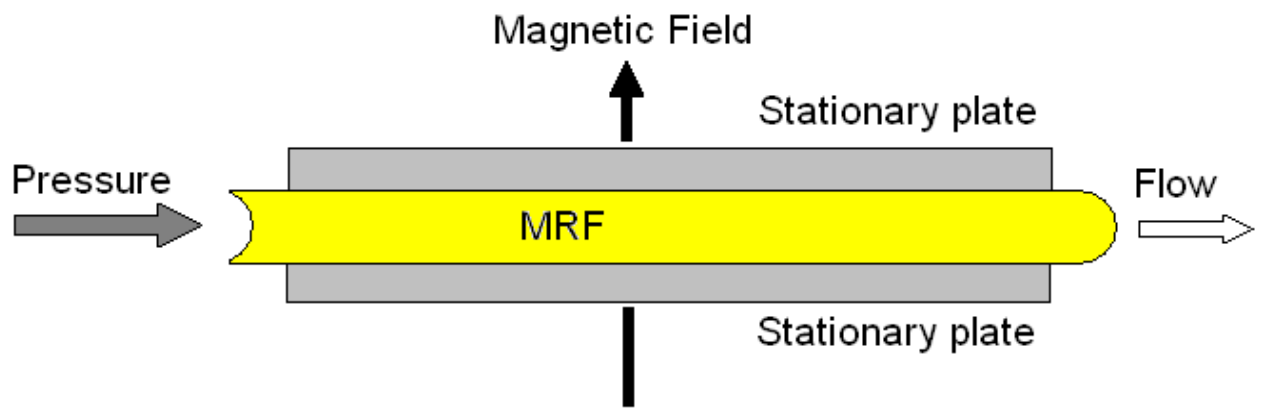

Fig. 3, Valve Mode 
The pressure drop created in this mode e.g. in a damper is the sum of the viscous (pure rheological) component $\Delta P_{r}$ and the magnetic field dependent (magnetorheological) component $\Delta P_{m r}$. The value of this pressure drop is defined using the following approximation [4]:

$$
\Delta P=\Delta P_{r}+\Delta P_{m r}=\frac{[12 \cdot \eta \cdot Q \cdot L]}{\left[g^{3} \cdot w\right]}+\frac{\left[f \cdot \tau_{m r} \cdot L\right]}{g}
$$

In the viscous component in this equation, $\eta[\mathrm{Pa} \cdot \mathrm{s}]$ is the dynamic viscosity, $Q\left[\frac{m^{3}}{s}\right]$ is the flow rate and $L, w, g[m]$ is the geometric length, width and gap size of the flow channel, and this part of the equation has a theoretical justification. In contrast the other part of the equation, the magnetic field dependent component, an empirical approach has been used, and here $\tau_{m r}\left[\frac{N}{\mathrm{~mm}^{2}}\right]$ is the yield stress developed in response to the applied magnetic field, and $L, g[m]$ are the same geometric data as in the rheological pressure drop part. The other factor $f[-]$ (no units) is an empirical factor and is determined experimentally. It is necessary to explain the difference between the observed pressure drop $\Delta P$ and the pressure drop calculated from rheological principles alone $\Delta P_{r}$. This is the pressure drop due to magnetorheological principles $\Delta P_{m r}$. It is clearly dependent on the yield stress developed in response to the applied magnetic field and to the above geometrical data, but there are also other factors which have an effect on this pressure drop, and the influence of these other factors are represented by the empirical factor $\mathrm{f}$. The factor is found experimentally to be dependent on the proportion of the purely rheological pressure drop to the total observed pressure drop.

The factor $\mathbf{f}$ takes:

The value 2 ( $\mathrm{f}=2$ ) in case of:

$$
\frac{\left[\Delta P_{m r}\right]}{\left[\Delta P_{r}\right]}<1
$$


and same factor $\mathbf{f}$ takes the value of $3(\mathrm{f}=3$ ) in case that:

$$
\frac{\left[\Delta P_{m y}\right]}{\left[\Delta P_{r}\right]} \sim 100
$$

Equation (6) can be used for the design of MRF applications in valve mode. Using this equation the minimum volume of active fluid can be established as:

$$
V=L . w . g=\left[\frac{12}{f^{2}}\right] \frac{\eta}{\tau^{2}}\left[\frac{\Delta P_{m r}}{\Delta P_{r}}\right] Q . \Delta P_{m r}
$$

This minimum volume of fluid is required to achieve a desired MRF effect at given flow rate $Q$ with the specified pressure drop.

The Rheonetic linear damper shown below is designed for use as a secondary suspension element in on- and off-highway vehicles [3]. This application is a damping control unit and is one of the first applications of MRF in the automotive industry. A magnetic coil integrated into the piston of the damper generates a magnetic field and this magnetic field regulates the MRF flow resistance within the damper. Figure 4 and Table 4 show the application features for this mode with reference to damper applications. 


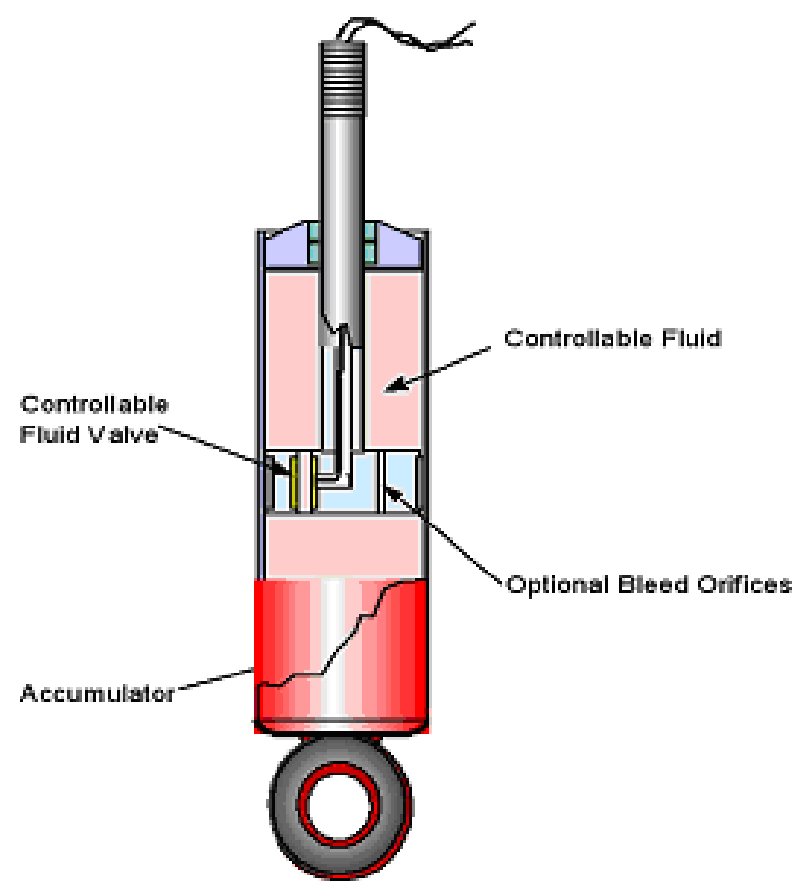

Fig. 4, Functional principle of MRF damper [1-5]

Table 4, Valve mode features [1-5]

\begin{tabular}{|l|l|}
\hline \multicolumn{1}{|c|}{ Application features } & Rheonetic $^{\mathrm{TM}}$ (seat suspension damper) \\
\hline Operational mode & Valve mode \\
\hline Shear stress level & $100 \mathrm{kPa}$ \\
\hline Shear rate level & $50.0001 / \mathrm{sec}$ \\
\hline Packaging & Diameter 35, length $100 \mathrm{~mm}$ \\
\hline Operational input energy & 4 Watts (1A, 4V) \\
\hline Power level in the application & $600 \mathrm{Watts}$ \\
\hline Active fluid volume & $0.3 \mathrm{ml}$ \\
\hline Total fluid volume & $50 \mathrm{ml}$ \\
\hline MRF Ratio (Fon / Foff) approx. & $2200 \mathrm{~N} / 400 \mathrm{~N}=5.5$ \\
\hline
\end{tabular}

Using this simple mechanical principle the damping arrangement becomes controllable and the vibration transmission and excitation frequency for a suspended seat can be adjusted accordingly. Proper choice of MRF parameters extended to seat suspension could eliminate any resonance problems and allow the system to be 
isolated from high frequency problems [3]. Since 2002 the valve mode is used in dampers in automotive vehicle suspensions manufactured by GM / Delphi.

These MRF applications bring additional functionality whilst keeping the simplicity. Other possible MRF applications using this mode are dampers for knee prosthesis, vibration dampers, seismic dampers for civil industry, active engine mounts and propshaft mounts.

\subsection{Shear Mode:}

The second operational mode is the direct shear mode. The direct shear mode is used in brakes and clutches and is shown schematically in Figure 5.

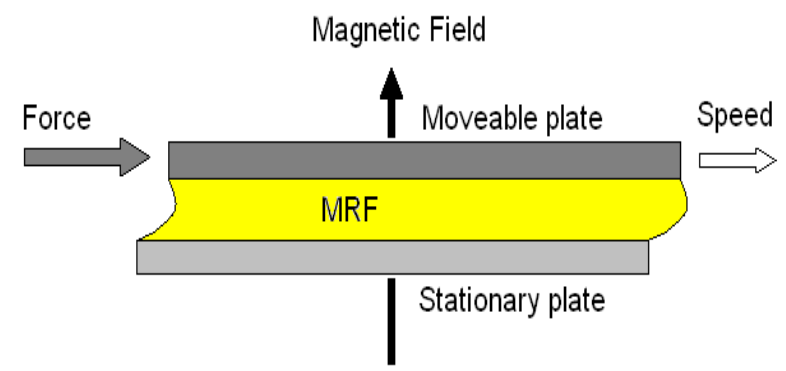

Fig. 5, Direct Shear Mode

The total force in the shear mode can be separated into a viscous (pure rheological) component $F_{r}$ and a magnetic field dependent (magneto-rheological) component $F_{m r}$. The total shear force is defined through the following approximation:

$$
F=F_{\gamma}+F_{m r}=\frac{[\eta \cdot S \cdot A]}{g}+\tau \cdot A
$$

In this equation $\eta[\mathrm{Pa} \cdot \mathrm{s}]$ is the dynamic viscosity, $S\left[\frac{\mathrm{m}}{\mathrm{s}}\right]$ is the relative speed, $A=L \cdot w$ is the working interface area and $L, w, g[m]$ are the length, width and gap size of the flow channel. In the magnetic field dependent component $\tau_{m r}\left[\frac{N}{\mathrm{~mm}^{2}}\right]$ is the 
yield stress developed in response to the applied magnetic field and $\mathrm{A}=\mathrm{Lw}$ is again the working interface area.

Equation (10) could be used for the design of MRF applications in direct shear mode. Using this equation the minimum volume of active fluid can be established:

$$
V=L \cdot w \cdot g=\left[\frac{\eta}{\tau^{2}}\right] \cdot\left[\frac{\left[F_{\gamma}\right]}{\left[F_{m r}\right]}\right] \cdot F_{m r} \cdot S
$$

This minimum volume of fluid is required to achieve a desired MRF effect $\left[\frac{F_{m r}}{F_{r}}\right]$ at a given speed S with the specified drag torque. This brake contains just a few parts: shaft, bearings, sealing devices, housing with coil, interface disc and MRF. The simplicity and easy control makes it a cost effective choice for controllable exercise equipment [3]. An MR fluid brake is currently being manufactured and sold as a controllable resistance element for programmable aerobic exercise equipment [3]. Figure 6 and Table 5 show some MRF features related to brake or clutch applications.

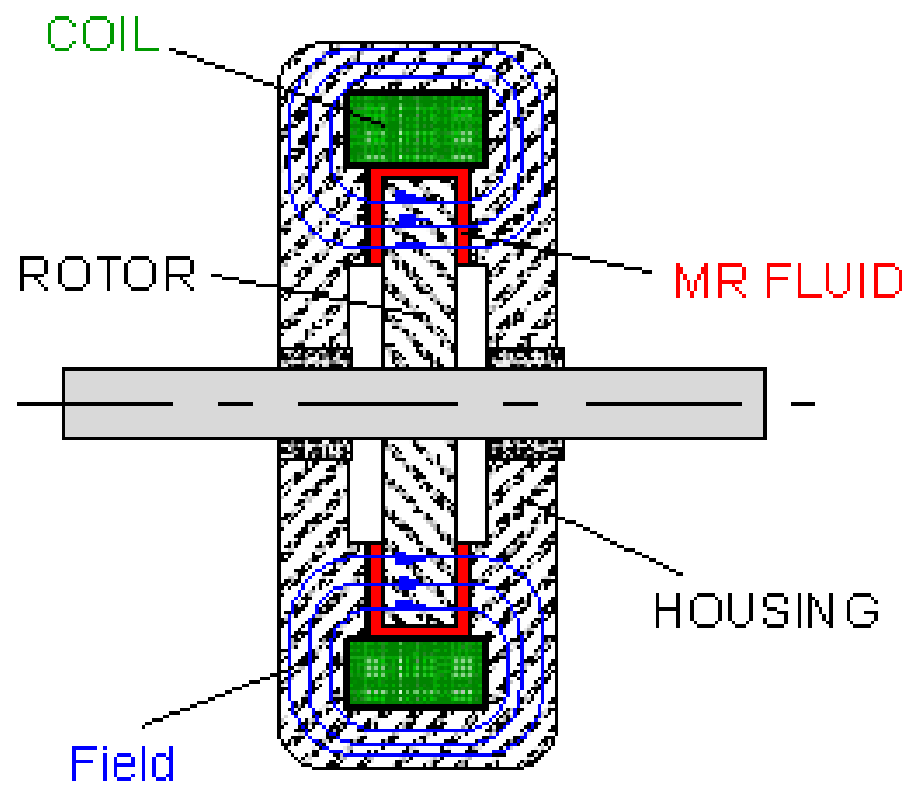

Fig. 6, Functional principle of MRF brake [1-5] 
Table 5, Shear mode features $[1-5,16]$

\begin{tabular}{|l|l|}
\hline \multicolumn{1}{|c|}{ Application features } & Rheonetic $^{\mathrm{TM}}$ (exercise brake) \\
\hline Operational mode & Shear mode or called direct-shear mode \\
\hline Shear stress level & $100 \mathrm{kPa}$ \\
\hline Shear rate level & $10.0001 / \mathrm{sec}$ \\
\hline Packaging & Diameter 92, length 35 mm \\
\hline Operational input energy & $\sim 10$ Watts $(0.8 \mathrm{~A}, 12 \mathrm{~V})$ \\
\hline Power level in the application & $700 \mathrm{Watts}$ \\
\hline Active fluid volume & $\sim 5 \mathrm{ml}$ \\
\hline Total fluid volume & $5 \mathrm{ml}$ \\
\hline MRF Ratio (Ton / Toff) approx. & $7 \mathrm{Nm} / 0.25 \mathrm{Nm}=28$ \\
\hline
\end{tabular}

The direct-shear mode and the valve mode have been studied in detail. Applications using these modes are today already present in many automotive products. The initially unexpected issue of in-use thickening has been solved. Good MR fluids show no measurable thickening in-use after more than 10 million cycles in the Motion MasterTM RD-10005 damper [2]. The degree of deterioration of the MR fluid depends on the application parameters like shear rate, temperature and duration of operation. The total amount of MRF dissipated energy, which can be controlled by the MRF unit, is defined by the following equation [2]:

$$
\text { Lifetime dissipated energy }(L D E)=\frac{1}{V} \cdot \int_{0}^{\text {Lifetime }} P \cdot d t
$$

where $V\left[\mathrm{~m}^{3}\right]$ is the total MR fluid volume in the application and $P[$ Watt $]$ is the mechanical power converted to heat in the MRF unit. The Lifetime Dissipated Energy "LDE" is the total mechanical energy dissipated per unit volume of MR fluid over the lifetime of the device [2]. Today's MRF applications are operating within the LDE range of approximately $3 \mathrm{MJ} / \mathrm{cm}^{3}$. If the LDE limit is exceeded, the MR fluid becomes thickened to a level where the MR effect is no longer significant enough to be considered as a controllable feature. The next steps in MRF development will be 
the conversions of various other conventional applications into devices using MR principles. There is also a need to develop fluids for the high shear regime from $10^{4} 1 / \mathrm{s}$ to $10^{6} 1 / \mathrm{s}$, with acceptable values of the LDE being higher than $10^{7} \mathrm{~J} / \mathrm{cm}^{3}$.

Observing patent databases and other information in the public domain it is clear that many feasibility studies are on-going where MR technology is being considered as a competitive technology for the future. Excellent features like simplicity, fast response, simple interface between electrical power input and mechanical power output using a magnetic field, and the controllability make the MRF technology the future technology for many applications. However some applications requirements are challenging the capabilities of the direct-shear mode and the valve mode, especially regarding the MRF-ratio. In some applications a higher ratio of the MRF effect is required to meet the specifications.

Some publications report that there is an additional operational mode feasible with a higher MRF effect than can be achieved with either the shear or the valve mode.

\subsection{Squeeze Mode:}

This third mode, called the squeeze mode, has not been studied so thoroughly comparing with the direct-shear mode and the valve mode. This mode of operation is shown schematically in Figure 7.

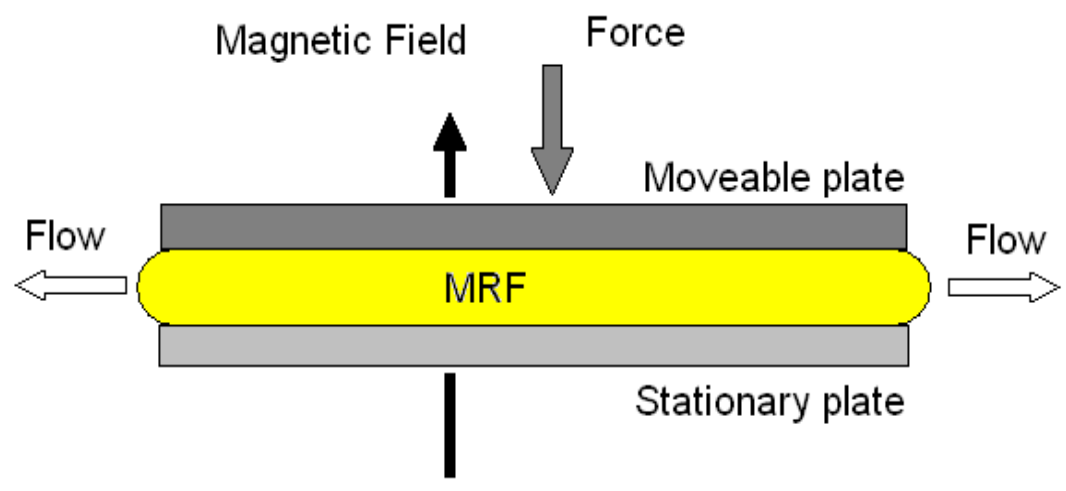

Fig. 7, MRF in squeeze mode 
Some small-amplitude vibration dampers use this mode. For small motions, this mode seems to offer the possibility of very large forces which can be controlled by the MRF effect [6]. In one of the most recent theoretical evaluations of the squeeze-strengthen effect in magneto-rheological fluids [15] the operation of this mode is described. It is suggested that a yield stress could be achieved which would be ten times as large as that which is possible with either the direct-shear or the valve mode. X.Z. Zhang evaluated theoretically in the reference [15] and confirmed with some experiments the higher capability of this specific operational mode. The higher yield stress under magnetic field means a higher ratio between on- and off- states. A stronger MRF effect in combination with advantages already described above would make MRF technology even more attractive and the technology of choice for the next generation of many more automotive and industrial applications.

\section{Conclusions:}

The Magneto-Rheological-Fluid (MRF) technology is one of the old "newcomers" coming to the market at high speed. The world is full of potential MRF applications. For every system where it is desirable to control motion using a fluid with changing viscosity, a solution based on MRF technology may be an improvement in functionality and costs. Simplicity and more intelligence in the functionality are key features of the MRF technology. Excellent features like fast response, simple interface between electrical power input and the mechanical power output, and controllability make MRF the next technology of choice for many applications. Direct shear mode (used in brakes and clutches) and valve mode (used in dampers) have been studied thoroughly and several products are already present in the market. Future developments include an increase in the acceptable lifespan of MRF devices, in terms of the total energy dissipated from the equipment throughout its working lifetime and fluids with higher shear regimes. 


\section{References:}

[1] Phillips, R.W., Engineering Applications of Fluids with a Variable Yield Stress, Ph.D. Thesis, University of California, Berkeley (1969)

[2] J.D. Carlson, What Makes A Good MR Fluid, $8^{\text {th }}$ International Conference on Electrorheological (ER) and Magnetorheological (MR) Suspensions, Nice (July 2001)

[3] J.D. Carlson, D.M. Catanzarite and K.A.St. Clair, Lord Corporation, Cary, NC 27511 USA, Commercial Magneto-Rheological Fluid Device, Proceedings of the 5th International Conference on ER Fluids, MR Fluids and Associated Technology, U. Sheffield, UK, pp. 20-28 (1995)

[4] M.R. Jolly, J.W. Bender and J.D. Carlson, Properties and Applications of Commercial Magnetorheological Fluids, Proceedings of the 5th SPIE Annual Int. Symposium on Smart Structures and Materials, San Diego, CA (1998).

[5] Lord Corporation, Dr. Dave's Do-It-Yourself MR Fluid, Designing with MR Fluid, Magnetic Circuit Design, FAQs, www.lord.com (2004)

[6] J.D. Carlson, Magnetorheological Fluid Actuators, Adaptronics and Smart Structures, Editor H. Janocha Springer Berlin 1999, Page 180-195, ISBN 3-54061484-2

[7] J. Huang, J.Q. Zhang, Y. Yang, Y.Q. Wei, Analysis and design of a cylindrical magnetorheological fluid brake, Journal of Materials Processing Technology 129 (2002), P 559-562

[8] P.L. Wong, W.A. Bullough, C. Feng, S. Lingard, Tribological performance of magnetorheological suspensions, Wear 247 (2001), Page 33-40

[9] D. Lampe, Untersuchungen zum Einsatz von Magnetorheologischen Fluiden in Kupplungen, Technische Universität Dresden, Ph.D. Thesis, Handout (2000) [10] D. Lampe, Anwendungen von Magnetorheologischen Fluiden in Kupplungen, Institut für Luft- und Raumfahrttechnik der Technische Universität Dresden, http://www.donnerflug.de/publikationen/antrieb.pdf

[11] Dr. V. Rabitschin, Einführung in die Rheologie, Kurs1 und Kurs2, University of Graz, www.copuserve.de/ctmattke/Kurs1.html, www.copuserve.de/ctmattke/Kurs1.html, [12] A. Milecki, Investigation and control of magneto-rheological fluid damper, International Journal of Machine and Manufacture 41 (2001), Page 379-391

[13] Magnetic fluid clutch, Technical News Bulletin, National Bureau of Standards, 32/4 (1948), Page 54-60

[14] W. Wislow, Field Responsive Fluid Couplings, US Pat. No. 2.886.151, (1959)

[15] X.Z.Zhang, X.L.Gong, P.Q.Zhang and Q.M. Wang, Study on the mechanism of the squeeze-strengthen effect in magnetorheological fluids, Journal of Applied Physics, Volume 96, No. 4, Page 2359-2364 (2004)

[16] J.D. Carlson, Lord Corporation, Cary, NC 27511 USA, MRF Workshop in

Carry, North Caroline, Workshop handouts (2004). 